\title{
Constraining Peptide Conformations with the Help of Ring-Closing Metathesis
}

\author{
Adrian Glas ${ }^{\mathrm{a}, \mathrm{b}}$ \\ Tom N. Grossmann*a,b \\ a Technical University Dortmund, Department of Chemistry \\ and Chemical Biology, Otto-Hahn-Straße 6, 44227 Dortmund, \\ Germany \\ b Chemical Genomics Centre of the Max Planck Society, Otto- \\ Hahn-Straße 15, 44227 Dortmund, Germany \\ tom.grossmann@cgc.mpg.de
}
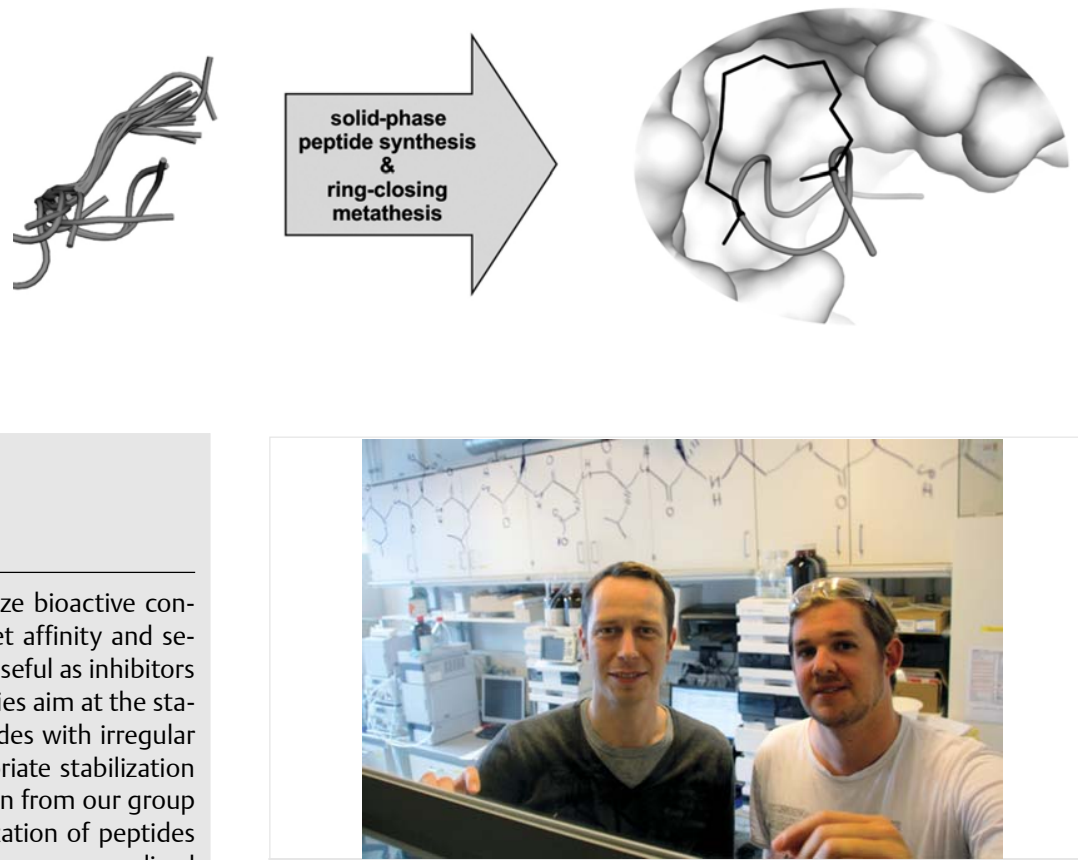

Accepted after revision: 14.10 .2014

Published online: 18.11 .2014

DOI: 10.1055/s-0034-1379425; Art ID: st-2014-p0673-sp

\begin{abstract}
Chemical modifications are used to stabilize bioactive conformations of peptides thereby increasing their target affinity and selectivity. Such modified peptides proved particularly useful as inhibitors of protein-protein interactions. Most of these strategies aim at the stabilization of $\alpha$-helices and $\beta$-sheets. The use of peptides with irregular secondary structure is hampered by a lack of appropriate stabilization approaches. Herein, we highlight a recent contribution from our group that uses ring-closing metathesis for the macrocyclization of peptides with irregular secondary structure. The peptide precursors are cyclized on solid support to provide cross-linked architectures that bind the human protein 14-3-3 thereby inhibiting its interaction with virulence factor exoenzyme $\mathrm{S}$ from Pseudomonas aeruginosa.
\end{abstract}

Key words cyclic peptides, hydrophobic cross-links, ring-closing metathesis, protein-protein interactions, Pseudomonas aeruginosa

For chemists, proteins can be a rich source of inspiration. In particular, structural information freely available via the protein data bank (PDB) ${ }^{1}$ provides a blueprint for the design of peptide-based bioactive molecules. Our group uses such information for the development of biocompatible chemical reactions ${ }^{2}$ and selective protein binders. ${ }^{3,4}$ Here, we explore the design of an inhibitor of a proteinprotein interaction (PPI). PPI play important roles in virtually all cellular processes, and their inhibition allows the manipulation of biological systems. ${ }^{5}$ This renders PPI inhibitors interesting compounds in chemical biology research and in drug development. ${ }^{6}$ The use of small-molecule PPI inhibitors is hampered by the fact that many PPI interfaces comprise large areas and lack pronounced pockets that are considered mandatory for small-molecule binding. ${ }^{5,7}$ Taking into consideration that the protein binding epitopes are defined by participating peptide sequences, peptide-based PPI inhibitors were envisioned. ${ }^{4,8,9}$ In solution, small peptides are highly flexible and adopt a defined bioactive configuration only upon binding. This leads to entropic penalty resulting in decreased affinity compared to the confor-
Tom N. Grossmann (left) is group leader at the Technical University and the Chemical Genomics Centre in Dortmund, Germany. His research interests involve the stabilization of peptide secondary structures and the use of biocompatible reactions. His lab is supported by the Emmy Noether program of the German Research Foundation (DFG). $\mathrm{He}$ is a chemist by training, and after performing undergraduate research in the lab of K. P. C. Vollhardt at the University of California Berkeley, he received his PhD under the guidance of Prof. O. Seitz at the Humboldt University Berlin in 2008. He then moved to Cambridge, USA for postdoctoral research in the group of Prof. G. L. Verdine at Harvard University until 2011.

Adrian Glas (right) was born in Berlin, Germany, where he studied chemistry at the Humboldt University, receiving his diploma in 2011. Since 2012 he is a PhD student in the Grossmann lab where he is working on the stabilization of irregular peptide secondary structures and their application as PPI inhibitors.

mationally constrained binding epitope within the originating protein. ${ }^{10}$ To constrain a peptide in its bioactive conformation various cyclization approaches have been developed. Early examples involve cyclization of $\mathrm{N}$ - and $\mathrm{C}-$ terminus leading to classic cyclic peptides..$^{11}$ Aiming for the inhibition of PPI, $\alpha$-helices have been stabilized (Figure 1) by side-chain-to-side-chain cross-links such as lactams $(\mathrm{A})^{12}$ and disulfides (B), ${ }^{13}$ or by ring-closing metathesis (RCM) of non-natural amino acids ( $C$ and D). ${ }^{14-16}$ Additional examples of peptide-derived PPI inhibitors involve $\beta$-turns cyclized by RCM $(E)^{17}$ and cyclic $\beta$-sheets stabilized by the incorporation of D-proline-L-proline turn structures $(F){ }^{18}$ 
A

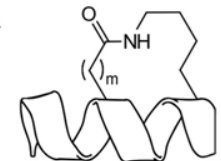

C

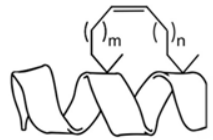

E

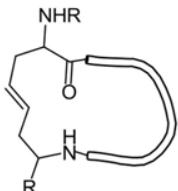

B
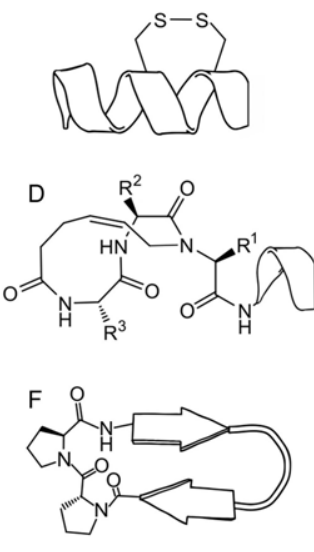

Figure 1 Stabilized secondary structure motifs used as PPI inhibitors. Stabilized $\alpha$-helixes: A) lactam; B) disulfide; C) hydrocarbon staple; D) hydrogen-bond surrogate (HBS); E) cyclic $\beta$-turn by RCM; F) cyclic $\beta$ sheet with D-proline-L-proline turn.

In particular, the stabilization of $\alpha$-helices has provided numerous peptide-based PPI inhibitors. ${ }^{4,9}$ However, the lack of strategies for the stabilization of irregular binding sequences, which frequently occur in PPI interfaces, ${ }^{19}$ limits the number of usable epitopes. Recently, our group in collaboration with the Ottmann lab (TU Eindhoven) reported a rational and interactive approach that allows the stabilization of irregular peptide secondary structures. ${ }^{3}$ Key hydrophobic residues crucially involved in protein binding were replaced by a lipophilic hydrocarbon cross-link that was formed in a RCM. Subsequent optimization of the linker architecture provided macrocyclic peptides which bind the

human adaptor protein 14-3-3 $\zeta$ with nanomolar affinities and inhibit its interactions with the virulence factor exoenzyme $S$ (ExoS) in vitro.

ExoS is a protein of Pseudomonas aeruginosa, a pathogenic gram-negative bacterium which is a major cause of health-care-associated infections and often shows multidrug resistances. Mutant studies showed that inhibition of the interaction between 14-3-3 and ExoS results in decreased infection rates. ${ }^{20}$ We decided to use an 11-mer peptide sequence (ESp) derived from the 14-3-3 binding motif of ExoS as a starting point for the development of corresponding PPI inhibitors. ESp was synthesized by Fmocbased solid-phase peptide synthesis (SPPS). Its binding affinity for $14-3-3 \zeta\left(K_{d}=1.14 \mu \mathrm{M}\right)$ was determined by fluorescence polarization (FP). In addition, the peptide was cocrystallized with $14-3-3 \zeta$ to confirm the binding mode earlier reported for the entire 14-3-3 binding motif of ExoS. Both, the synthetic peptide and the published binding motif, bind $14-3-3 \zeta$ in an irregular and mostly extended conformation, and their corresponding residues superimpose almost perfectly $(\mathrm{rmsd}=0.171 \AA)^{20}$

The use of peptide stabilization approaches such as cyclization of $\mathrm{N}$ - and C-terminus or hydrogen-bond surrogates (HBS) were discarded due to the elongated structure of ESp. Independent of the macrocyclic architecture, previous examples aimed at the replacement of residues that are not involved in binding. Notably, in the case of ESp almost all residues directly interact with $14-3-3 \zeta$, which complicates the introduction of a constraining element. We noticed that three leucines (L422, L423, L426) and one alanine (A425) exhibit side-chain arrangements which should, according to distance and orientation, allow cross-linking. Since, these residues form crucial nonpolar interactions

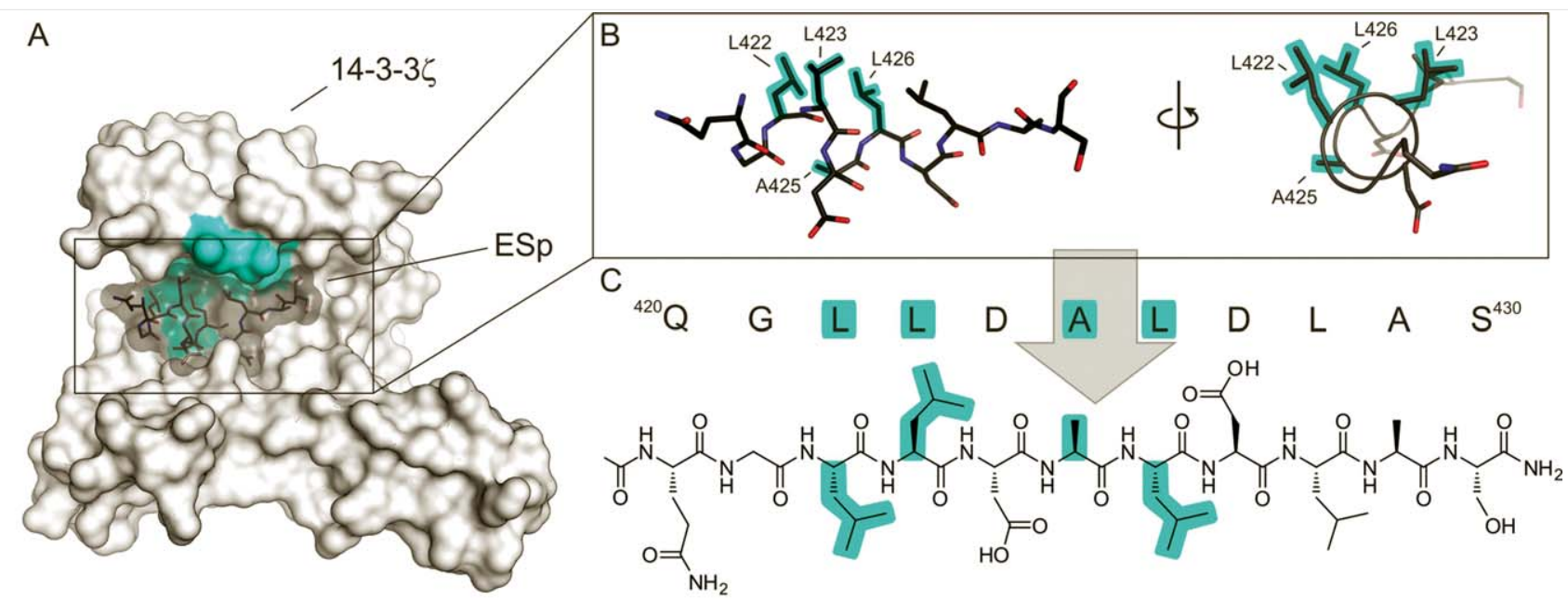

Figure 2 A) Crystal structure of 14-3-3Z in complex with ESp (sticks: black = C, red = O, blue = N; PDB: 4N7G). B) Left: Side view of ESp in stick representation. Right: Front view of ESp (residues in stick representation, backbone in ribbon presentation; sticks: red = O, dark blue = N). C) Sequence of ESp in one-letter amino acid code with chemical structure; important hydrophobic residues are highlighted in turquois. 
E S P Q G L L D A D L A S<smiles></smiles>

$i \quad i+4$

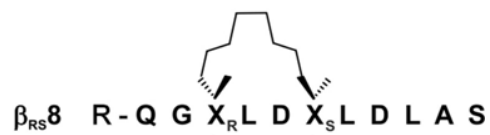

$i \quad i+3$

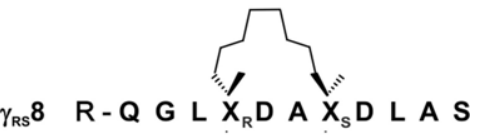

i $\quad i+3$

Figure 3 First set of peptides with eight carbon cross-links at three different positions $(\alpha, \beta, \gamma)$ with corresponding absolute configuration of non-natural amino acids [ $\mathrm{X}_{\mathrm{S}}=\mathrm{S}$-configured; $\mathrm{X}_{\mathrm{R}}=R$-configured;

$\mathrm{R}=$ acetyl group or a polyethylene glycol spacer (PEG2), capped with fluorescein isothiocyanate (FITC)].

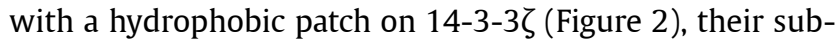
stitution with a lipophilic cross-link was envisioned. This would allow $\mathrm{A}$ ) the preservation of the hydrophobic character of the 14-3-3 binding motif of ESp and B) the introduction of a constraining element. Based on the crystal structure of ESp in complex with 14-3-3 $\zeta$, three peptides, each cross-linked with a $\left(\mathrm{CH}_{2}\right)_{8}$-bridge, were designed $\left(\alpha_{S S} 8\right.$, $\beta_{\mathrm{RS}} 8, \gamma_{\mathrm{RS}} 8$; Figure 3).

In analogy to stapled peptides, ${ }^{21}$ depending on their relative spacing, different absolute configurations of the unnatural amino acids were chosen: For a spacing of $i, i+4$ two $S$-configured amino acids $(\alpha)$, and for $i, i+3$ an $R$-configuration at position $i$ and an $S$-configuration at position $i+3(\beta$, $\gamma){ }^{16,22}$ We decided to install the hydrophobic bridges via olefin RCM. The metathesis reaction provides high yields under mild conditions and tolerates the functional groups present in the protected peptide. ${ }^{14,21}$

For the introduction of the hydrocarbon cross-link, selected residues were substituted by $\alpha$-methyl, $\alpha$-alkenyl non-natural amino acids. Synthesis of the peptide sequence was performed by Fmoc-based SPPS (Figure 4). Subsequent cross-linking via RCM used first-generation Grubbs catalyst with solid-phase-bound, protected peptides. To avoid diastereomeric mixtures due to the formation of $E$ - and Z-configured double bonds, the generated olefin was reduced with 2,4,6-triisopropylbenzenesulfonyl hydrazide (TPSH). Reaction yields were monitored by analyzing cleaved aliquots via mass spectrometry-coupled high-performance liquid chromatography. For FP-based affinity assays, peptides were coupled with a polyethylene glycol spacer (PEG2, derived from 8-amino-3,6-dioxaoctanoic acid) and capped

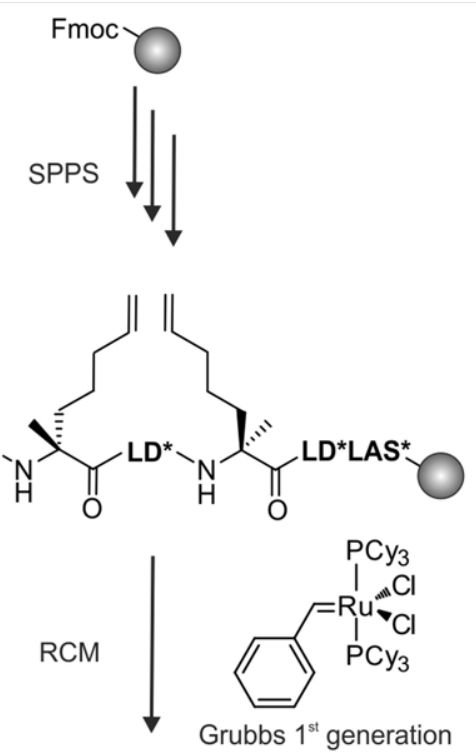

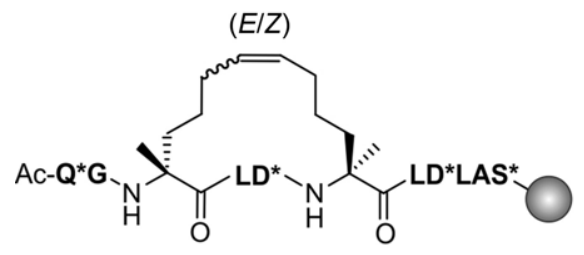<smiles>CC(C)c1cc(C(C)C)c(S(=O)(=O)NN)c(C(C)C)c1</smiles>

Figure 4 Synthetic route towards $\beta_{\mathrm{RS}} 8$. Olefinic, non-natural amino acids were introduced by SPPS. Natural amino acids are shown in bold; ${ }^{*}=$ protecting group. Ruthenium-catalyzed RCM was performed with firstgeneration Grubbs catalyst at room temperature in 1,2-dichlorethane (DCE). Resulting double bonds were reduced with 2,4,6-triisopropylbenzenesulfonyl hydrazide (TPSH) at $50{ }^{\circ} \mathrm{C}$ in $\mathrm{N}$-methyl-2-pyrrolidone/piperidine. Reactions were followed by test cleavages of peptides (C-terminal amide) and analyzed by RP-HPLC ESI-MS of test-cleaved peptide.

with fluorescein isothiocyanate (FITC). After synthesis and HPLC purification, affinities of the initial three peptides for 14-3-3 $\zeta$ were determined. Peptides harboring the crosslink in $\alpha$ - and $\gamma$-position, respectively, bind $14-3-3 \zeta$ with an about 20 -fold lower affinity $\left(K_{\mathrm{d}}=\mathrm{ca} .20 \mu \mathrm{M}\right)$ compared to $\operatorname{ESp}\left(K_{\mathrm{d}}=1.1 \mu \mathrm{M}\right)$. A 4.6-fold increase in affinity $\left(K_{\mathrm{d}}=0.25\right.$ $\mu \mathrm{M}$ ) was observed for the $\beta$-cross-linked peptide $\beta_{\mathrm{RS}} 8$. Con- 
sidering that the cross-link substitutes two residues that are crucially involved in protein binding, this is a remarkable result.

To confirm the binding site and to provide a structural basis for a subsequent optimization of cross-linked peptide $\beta_{\mathrm{RS}} 8$, cocrystallization with $14-3-3 \zeta$ (aa 1-230) and X-ray crystallography were performed. While the C-terminus of $\beta_{\mathrm{RS}} 8$ superimposes with ESp, a dislocation of the $\mathrm{N}$-terminal part of the backbone is observed (data not shown). This leads to the absence of polar contacts between $\beta_{\mathrm{RS}} 8$ and 14$3-3 \zeta$ that were present for ESp. In addition, the interaction between leucine 423 and $14-3-3 \zeta$ is missing. Inspection of the macrocycle in peptide $\beta_{\mathrm{RS}} 8$ indicates that the length of the cross-link should significantly influence binding affinity. We also noticed that the inversion of the configuration of the non-natural amino acid at position 422 may allow a different spatial orientation of the entire cross-link.

With the intention to design more affine binders, a small library of peptides with altered cross-link length and/or inverted stereochemistry at the $C \alpha$ of amino acid position 422 was synthesized (Figure 5,A). For peptides with unchanged absolute configuration (Figure 5,A, line 26) neither shortened nor extended cross-links resulted in improved affinities for 14-3-3 3 . Interestingly, the series of epimers with an $R$ to $S$ inversion at position 422 (Figure 5,A, line 7-13) provide a clear structure-affinity relationship with an optimum linker length of $12 \mathrm{CH}_{2}$ groups $\left(\beta_{\mathrm{SS}} 12\right.$, $K_{\mathrm{d}}=41 \mathrm{nM}$ ). Compared to the initial peptide $\beta_{\mathrm{RS}} 8$ this accounts for sixfold, and compared to the unmodified starting peptide ESp for 27-fold more affine binding of $14-3-3 \zeta$. To understand the molecular details of $14-3-3 \zeta$ recognition by $\beta_{\mathrm{SS}} 12$, the cocrystal structure was determined (Figure 5,B).
The cross-link shows strong engagement in hydrophobic interactions with 14-3-3 $\zeta$ (Figure 5,C). In addition, the modified architecture allows the backbone to arrange very similar to the wild-type peptide ESp. This recovers both, the interaction of leucine 423 and water mediated polar contacts which are absent in $\beta_{\mathrm{RS}} 8$ due to a rearrangement of the backbone.

To evaluate the thermodynamic effects caused by macrocyclization more closely, isothermal titration calorimetry (ITC) was performed for the complexes of 14-3-3 $\zeta$ with ESp, $\beta_{\mathrm{RS}} 8$, and $\beta_{\mathrm{SS}} 12$, respectively (Figure 5,D). In general, the ITC-based affinities reflect the results obtained by FP. The Gibbs free energy $(\Delta \mathrm{G}=\Delta \mathrm{H}-\mathrm{T} \Delta \mathrm{S})$ for ESp-14-3-3 $\zeta$ complex formation is almost exclusively determined by binding enthalpy $(\Delta \mathrm{H})$. Only negligible entropic contributions $(-\mathrm{T} \Delta \mathrm{S})$ were observed. This may be caused by compensation of the hydrophobic effect (which supports the interaction) by entropic penalties due to conformational restrictions upon binding. In contrast to ESp both macrocyclic peptides $\beta_{\mathrm{RS}} 8$ and $\beta_{\mathrm{SS}} 12$ show significant entropic contributions. A possible explanation for this behavior is that the unbound macrocyclic peptides experience a conformational constrain, reducing entropic penalty upon binding. To test this hypothesis we performed circular dichroism (CD) and ${ }^{1} \mathrm{H}$ NMR spectroscopy. Both measurements indicate a rigidified conformational character for both macrocyclic free peptides.

Based on ITC experiments, the binding enthalpy of $\beta_{\mathrm{RS}} 8$ is reduced by more than $50 \%$ when compared to ESp. For $\beta_{\mathrm{SS}} 12$ on the other hand, most of this enthalpy is restored, which might be explained by a similar backbone configuration in ESp and $\beta_{\mathrm{SS}} 12$ supporting more extensive polar interactions with $14-3-3 \zeta$ than for $\beta_{\mathrm{RS}} 8$. In conclusion, the
A

\begin{tabular}{|c|c|c|c|c|}
\hline & Peptide & Sequence & $\left(\mathrm{CH}_{2}\right)_{x}$ & $K_{\mathrm{d}}[\mathrm{mM}]$ \\
\hline 1 & ESp & QG L LDA LDLAS & & $1.14 \pm 0.05$ \\
\hline 2 & $\beta_{\mathrm{RS}} 7$ & $\mathrm{QG} 4_{R} \mathrm{LD}_{S}$ LDLAS & 7 & $6.5 \pm 0.5$ \\
\hline 3 & $\beta_{\mathrm{RS}}{ }^{8}$ & QG4 ${ }_{R}$ LD4 $4_{S}$ LDLAS & 8 & $0.25 \pm 0.01$ \\
\hline 4 & $\beta_{\mathrm{RS}}{ }^{9}$ & QG4 ${ }_{R}$ LD $5_{S}$ LDLAS & 9 & $2.0 \pm 0.1$ \\
\hline 5 & $\beta_{\mathrm{RS}} 10$ & QG4 $4_{R}$ LD6 $6_{S} L D L A S$ & 10 & $0.65 \pm 0.02$ \\
\hline 6 & $\beta_{\mathrm{RS}}{ }^{11}$ & QG6 ${ }_{R}$ LD 5 5 LDLAS & 11 & $0.99 \pm 0.03$ \\
\hline 7 & $\beta_{S_{S}}{ }^{7}$ & QG3SLD4SLDLAS & 7 & $>100$ \\
\hline 8 & $\beta_{S S}{ }^{8}$ & QG4SLD4SLDLAS & 8 & $1.5 \pm 0.3$ \\
\hline 9 & $\beta_{\text {SS }} 9$ & QG6 ${ }_{S}$ LD 3 ${ }_{S}$ LDLAS & 9 & $5.8 \pm 0.2$ \\
\hline 10 & $\beta_{\mathrm{SS}} 10$ & QG6SLD4SLDLAS & 10 & $0.36 \pm 0.02$ \\
\hline 11 & $\beta_{\mathrm{SS}} 11$ & QG5 $5_{S}$ LD6 $6_{S}$ LDLAS & 11 & $0.27 \pm 0.01$ \\
\hline 12 & $\beta_{\text {SS }} 12$ & QG6 $6_{S}$ LD6 $6_{S}$ LDLAS & 12 & $0.041 \pm 0.003$ \\
\hline 13 & $\beta_{\text {SS }} 13$ & QG6 ${ }_{S}$ LD $7_{S}$ LDLAS & 13 & $0.52 \pm 0.03$ \\
\hline
\end{tabular}

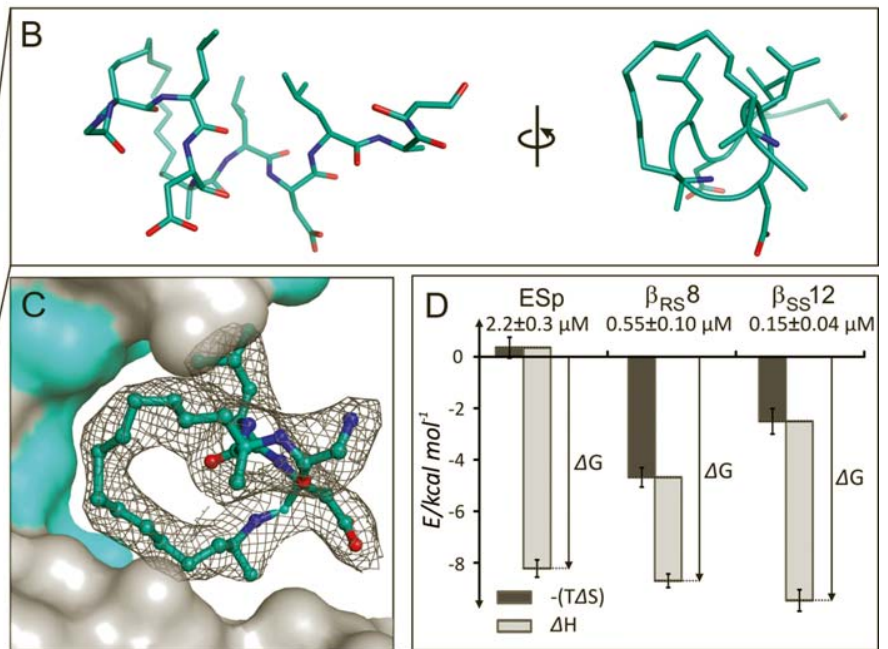

Figure 5 A) List of peptides (with C-terminal amide) including their sequence, cross-link length, and determined $K_{d}$ values. B) Side and front view of $\beta_{\mathrm{sS}} 12$ in stick representation (sticks: red $=\mathrm{O}$, dark blue $=\mathrm{N}$; PDB: $4 \mathrm{~N} 84$ ). $\mathrm{C}$ ) Final $2 \mathrm{~F}_{\mathrm{o}}-\mathrm{F}_{\mathrm{c}}$ electron density (contoured at $\left.1 \sigma\right)$ around the cross-link. Hydrophobic patch of $14-3-3 \zeta$ is highlighted in turquois. D) Comparison of ITC-derived enthalpic ( $\Delta \mathrm{H}$, light grey) and entropic contributions (-T $\triangle \mathrm{S}$, dark grey) to the free binding enthalpy $\left(\Delta \mathrm{G}\right.$, black arrow in y-axis) at $30^{\circ} \mathrm{C}$. 
constrained conformational freedom in $\beta_{\mathrm{SS}} 12$ in combination with extended hydrophobic and polar interactions

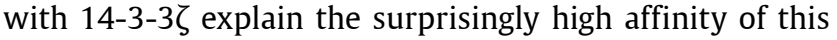
macrocylcic peptide for its receptor $14-3-3 \zeta$. $\beta_{\mathrm{SS}} 12$ also proved to be effective in inhibiting the interaction between $14-3-3 \zeta$ and a fragment of the virulence factor ExoS in vitro.

The introduction of constraints to conserve bioactive conformations is an important feature of peptide-based PPI inhibitors that were derived from protein sequences. While various approaches allow the stabilization of $\alpha$-helices and $\beta$-sheets, the use of irregular secondary structure motifs is limited by a lack of appropriate stabilization strategies so far. We presented the first example of such a rationally designed constrained peptide using the synthetic potential of the metathesis reaction. This strategy is appealing for the generation of new peptide-derived inhibitors, knowing that irregular structures participate widely in PPI. In addition, these findings A) expand the applicability of olefin ringclosing metathesis in SPPS, B) provide new biophysical insights into the influence of conformational constraints on peptide-protein recognition, and C) expand the scope of bioactive macrocyclic molecules. We believe that the expansion of the toolbox for peptide modifications will lead to compounds with improved binding characteristics and pharmacological behavior. Both are prerequisites for a more extensive use of peptides in drug development.

\section{Acknowledgment}

We thank the Fonds der Chemischen Industrie for financial support. TNG thanks the German Research Foundation (DFG, Emmy Noether program GR3592/2-1). This work was supported by AstraZeneca, Bayer CropScience, Bayer HealthCare, Boehringer Ingelheim, Merck KGaA, and the Max Planck Society.

\section{References}

(1) Berman, H. M.; Westbrook, J.; Feng, Z.; Gilliland, G.; Bhat, T. N.; Weissig, H.; Shindyalov, I. N.; Bourne, P. E. Nucleic Acids Res. 2000, 28, 235.
(2) Brauckhoff, N.; Hahne, G.; Yeh, J. T.; Grossmann, T. N. Angew. Chem. Int. Ed. 2014, 53, 4337.

(3) Glas, A.; Bier, D.; Hahne, G.; Rademacher, C.; Ottmann, C.; Grossmann, T. N. Angew. Chem. Int. Ed. 2014, 53, 2489.

(4) Spiegel, J.; Cromm, P. M.; Itzen, A.; Goody, R. S.; Grossmann, T. N.; Waldmann, H. Angew. Chem. Int. Ed. 2014, 53, 2498.

(5) Thiel, P.; Kaiser, M.; Ottmann, C. Angew. Chem. Int. Ed. 2012, 51, 2012.

(6) (a) Milroy, L. G.; Grossmann, T. N.; Hennig, S.; Brunsveld, L.; Ottmann, C. Chem. Rev. 2014, 114, 4695. (b) Hahne, G.; Grossmann, T. N. Bioorg. Med. Chem. 2013, 21, 4020.

(7) Wilson, A. J. Chem. Soc. Rev. 2009, 38, 3289.

(8) London, N.; Raveh, B.; Movshovitz-Attias, D.; Schueler-Furman, O. Proteins Struct. Funct. Bioinf. 2010, 78, 3140.

(9) Verdine, G. L.; Walensky, L. D. Clin. Cancer Res. 2007, 13, 7264.

(10) Houk, K. N.; Leach, A. G.; Kim, S. P.; Zhang, X. Y. Angew. Chem. Int. Ed. 2003, 42, 4872.

(11) Kessler, H. Angew. Chem., Int. Ed. Engl. 1982, 21, 512.

(12) Bracken, C.; Gulyas, J.; Taylor, J. W.; Baum, J. J. Am. Chem. Soc. 1994, 116, 6431.

(13) Jackson, D. Y.; King, D. S.; Chmielewski, J.; Singh, S.; Schultz, P. G. J. Am. Chem. Soc. 1991, 113, 9391.

(14) Blackwell, H. E.; Grubbs, R. H. Angew. Chem. Int. Ed. 1998, 37, 3281.

(15) (a) Grossmann, T. N.; Yeh, J. T.; Bowman, B. R.; Chu, Q.; Moellering, R. E.; Verdine, G. L. Proc. Natl. Acad. Sci. U.S.A. 2012, 109, 17942. (b) Patgiri, A.; Jochim, A. L.; Arora, P. S. Acc. Chem. Res. 2008, 41, 1289.

(16) Schafmeister, C. E.; Po, J.; Verdine, G. L. J. Am. Chem. Soc. 2000, 122, 5891.

(17) Miller, S. J.; Blackwell, H. E.; Grubbs, R. H. J. Am. Chem. Soc. 1996, $118,9606$.

(18) Fasan, R.; Dias, R. L.; Moehle, K.; Zerbe, O.; Obrecht, D.; Mittl, P. R.; Grutter, M. G.; Robinson, J. A. ChemBioChem 2006, 7, 515.

(19) Guharoy, M.; Chakrabarti, P. Bioinformatics 2007, 23, 1909.

(20) Ottmann, C.; Yasmin, L.; Weyand, M.; Veesenmeyer, J. L.; Diaz, M. H.; Palmer, R. H.; Francis, M. S.; Hauser, A. R.; Wittinghofer, A.; Hallberg, B. EMBO J. 2007, 26, 902.

(21) Kim, Y. W.; Grossmann, T. N.; Verdine, G. L. Nat. Protoc. 2011, 6, 761.

(22) Kim, Y. W.; Kutchukian, P. S.; Verdine, G. L. Org. Lett. 2010, 12, 3046. 